\title{
EDITORIAL
}

\section{Multiplex, megaplex, index, and complex: the present and future of laboratory diagnostics in rheumatology}

\author{
Mark H Wener* \\ See related research by Chandra et al., http://arthritis-research.com/content/13/3/R102
}

\begin{abstract}
In a recent issue of Arthritis Research \& Therapy, Chandra and colleagues described the use of multiple multiplex immunoassays and complex computer algorithms to investigate the possibility of improved laboratory diagnosis and novel classification of rheumatoid arthritis on the basis of biomarkers. Such complex predictive tools in rheumatology can be guided by the experience of multiplex testing in oncology, which has demonstrated the importance of uniform specimen handling and prospectively collected specimen repositories. Although there are high expectations for these complex approaches, they require careful evaluation.
\end{abstract}

Multiplex diagnostic testing refers to the use of laboratory devices that perform many assays on the same specimen at the same time with a single laboratory platform. In some respects, autoimmune rheumatic disease tests have long involved multiplexing. For example, the immunofluorescence test for antibodies to nuclear antigens (ANAs) detects multiple ANA patterns and specificities. Modern multiplex methodology typically involves suspensions of antigen-coated microscopic beads that are distinguishable by physical characteristics such as fluorescent color. Research multiplex assays may employ planar arrays of antigens spotted on glass slides. Panels of automated multiplex clinical laboratory tests are available for autoantibody testing, such as testing for the specificities of ANAs. Multiplex research assays are widely used to test for cytokines and other biomarkers. In

*Correspondence: wener@uw.edu

Department of Laboratory Medicine and Rheumatology Division, Department of Medicine, University of Washington, Box 357110, Seattle, WA 98195, USA a recent issue of Arthritis Research \& Therapy, Chandra and colleagues [1] explored the use of multiple multiplex assays (a 'megaplex'?) in the evaluation and categorization of patients with rheumatoid arthritis (RA).

Implementing high-quality multiplexed protein assays has many technical analytical challenges, including problems of uniformity, cross-reactivity, and optimization [2,3]. Rheumatoid factors and other anti-reagent antibodies may interfere [4], as, indeed, they can interfere with individual immunoassays. Ideally, each individual assay included in the multiplex assay should be evaluated fully. Other criteria for evaluating reports of multiplex assays are suggested in Table 1.

How should multiplex laboratory data be interpreted? When the biomarker number is small and their clinical value is understood, results can be sent to the provider directly for interpretation. However, access to multiple test results supports the creation of predictors that are more complex and hopefully more accurate, since they are based on the integrated results from more parameters.

Diagnostic algorithms using multiple biomarkers and complex multivariate data analysis have been investigated the most in oncology. Some oncologists express concern about a pattern of false discoveries in this field $[5,6]$. Some of the concerns raised by the oncology studies are relevant to rheumatology. One important message is that differences in the 'pre-analytical' phase of testing (that is, factors such as phlebotomy conditions and storage) can cause differences in laboratory test results, which may be magnified and masked by complex computations, and lead to incorrect conclusions. Measurement of some cytokines, hormones, and other biomarkers is known to be influenced by specimen handling [5,7]. In some ovarian cancer studies, initially exciting findings were determined to be caused not by the diagnosis but by different specimen handling by the institutions where the specimens originated [8]. What this points out is the importance of uniform specimen handling. It has been proposed that biomarker discovery and evaluation rely 


\section{Table 1. Criteria for evaluating multiplex assays}

1. Analytical performance parameters (for example, precision, analytical sensitivity, and linearity) of assays should be available for each analyte.

2. The clinical performance (clinical sensitivity and specificity) of each analyte within the multiplex should be comparable to that of assays for individual analytes.

3. The time required to produce all results of multiplex assay should be less than the sum of time required to produce results of individual assays.

4. The combination of multiplex assays should be appropriate for answering clinical questions; that is, the combinations of analytes measured within multiplex assay should make clinical sense.

on prospectively and uniformly collected specimens that include the range of subjects likely to be tested clinically, followed by blinded retrospective laboratory testing of sera from patients and controls randomly selected from the original prospective cohort. This so-called PRoBE (prospective specimen collection, blinded evaluation retrospectively) approach strengthens biomarker discovery and validation [9]. Establishing multipurpose population-based repositories may be necessary for definitive biomarker studies.

Many of the hopes and challenges of computer-assisted multiplex laboratory diagnostics are demonstrated in the paper by Chandra and colleagues. The authors used 7 different arrays to measure 41 conventional and novel autoantibodies and biomarkers. In addition, the authors used a multiplex bead assay to measure 13 selected cytokines and chemokines. Sera from 120 patients with RA, 55 rheumatic disease control patients, and 25 healthy individuals were tested. Over 1,100 RA sera were used to compare the analytical performance of novel and conventional immunoassays for rheumatoid factor and Creactive protein, and precision data were provided for 4 biomarkers.

The studies have the potential to be confounded by the demographic and pre-analytic specimen handling issues described previously: the sera from patients with RA came from one repository, the sera from spondylitis and psoriatic arthritis control patients each came from different sites, and the normal control sera were from another site. Differences between prolactin concentrations in different diagnostic groups were observed in this study, as in some previous ovarian cancer populations in which serum prolactin differences were explained by differences in specimen handling.

The computerized cluster analysis in the paper by Chandra and colleagues suggested that RA patients could be subdivided by distinct biomarker profiles. The US Food and Drug Administration (FDA) has used the term 'in vitro diagnostic multivariate index assay' (IVDMIA) to describe laboratory assays that combine the values of multiple individual tests into a single result or index that is not transparent and cannot be easily verified by the

\section{Table 2. Criteria for evaluating multivariate index assays}

1. Normal control and disease control groups used to generate the index should be matched demographically (age, gender, race, and geography) with the target group.

2. Pre-analytical variables (specimen type, specimen handling, and specimen storage) should be equivalent in control and diseased groups.

3. There should be a high ratio of subjects (patients) to measured analytes used to generate the index.

4. The accuracy (clinical sensitivity and specificity) of the index test should be tested and reported on the basis of populations of subjects (the 'test set' of diseased patients and controls) independently of the subjects (the 'training set') used to generate the index formulae or calculations.

5. The clinical accuracy (clinical sensitivity and specificity) of the index test should be compared with the accuracy of the most accurate of the individual analytes within the index or with the best available single diagnostic laboratory test or both.

provider [10], and the cluster analysis by the authors fits into that category. Although the FDA has yet to produce definitive guidelines for the evaluation of IVDMIAs, the suggestion is that tests of high complexity with high stakes for diagnosis or treatment (or both) will require proof of efficacy before being approved for clinical use. Some suggested criteria for evaluating reports of IVDMIAs are included in Table 2. One of the suggested criteria, which is common to all multivariate approaches, is that the ratio of cases of interest used to generate the model should be high relative to the number of predictor variables (a ratio of 10:1 to 20:1 has been suggested as a general rule) in order to have confidence in the results [11].

In the end, did the measurement of 54 biomarkers by Chandra and colleagues lead to improved diagnostic performance? From the 54 initial biomarkers, combinations of 6 tests (including 3 anti-citrullinated peptide assays) had the best diagnostic discrimination. However, the combinations performed no better than anti-CCP (anticyclic citrullinated protein antibodies) alone.

Where is rheumatology laboratory testing in the search for multiplex and multivariate approaches to diagnosis and stratification? Multiplex testing for ANA and ANCA (anti-neutrophil cytoplasmic antibodies) specificities is expanding. Multivariate assay approaches for lupus, RA, and other rheumatic diseases are being investigated. Whether or not these multivariate approaches lead to clinically significant and cost-effective improvement in testing remains to be seen, but, appropriately, the search is on. Chandra and colleagues are to be congratulated for their exploratory studies.

\section{Abbreviations}

ANA, antibody to nuclear antigens; FDA, US Food and Drug Administration; IVDMIA, in vitro diagnostic multivariate index assay; RA, rheumatoid arthritis.

\section{Competing interests}

MHW's laboratory at the University of Washington performs selected autoimmune laboratory testing and participates in preparation of teaching material for BioRad Laboratories. 
Published: 24 November 2011

\section{References}

1. Chandra PE, Sokolove J, Hipp BG, Lindstrom TM, Elder JT, Reveille JD, Eberl H, Klause U, Robinson WH: Novel multiplex technology for diagnostic characterization of rheumatoid arthritis. Arthritis Res Ther 2011, 13:R102.

2. Ellington AA, Kullo IJ, Bailey KR, Klee GG: Antibody-based protein multiplex platforms: Technical and operational challenges. Clin Chem 2010, 56:186-193.

3. Kricka LJ, Master SR: Quality control and protein microarrays. Clin Chem 2009, 55:1053-1055.

4. Todd DJ, Knowlton N, Amato M, Frank MB, Schur PH, Izmailova ES, Roubenoff R, Shadick NA, Weinblatt ME, Centola M, Lee DM: Erroneous augmentation of multiplex assay measurements in patients with rheumatoid arthritis due to heterophilic binding by serum rheumatoid factor. Arthritis Rheum 2011, 63:894-903.

5. Ransohoff DF, Gourlay ML: Sources of bias in specimens for research about molecular markers for cancer. J Clin Oncol 2010, 28:698-704.

6. Bossuyt PM: The thin line between hope and hype in biomarker research. JAMA 2011, 305:2229-2230.

7. Zhou X, Fragala MS, McElhaney JE, Kuchel GA: Conceptual and methodological issues relevant to cytokine and inflammatory marker measurements in clinical research. Curr Opin Clin Nutr Metab Care 2010, 13:541-547.
8. Thorpe JD, Duan X, Forrest R, Lowe K, Brown L, Segal E, Nelson B, Anderson GL, McIntosh M, Urban N: Effects of blood collection conditions on ovarian cancer serum markers. PLoS One 2007, 2:e1281.

9. Pepe MS, Feng Z, Janes H, Bossuyt PM, Potter JD: Pivotal evaluation of the accuracy of a biomarker used for classification or prediction: standards for study design. J Natl Cancer Inst 2008, 100:1432-1438.

10. Center for Biologics Evaluation and Research, Food and Drug Administration: Draft guidance for industry, clinical laboratories, and FDA staff. In vitro diagnostic multivariate index assays. Rockville, MD: Office of

Communication, Training and Manufacturers Assistance, HFM-40, Center for Biologics Evaluation and Research, Food and Drug Administration; 2007. Report 1610 [http://www.fda.gov/downloads/MedicalDevices/ DeviceRegulationandGuidance/GuidanceDocuments/ucm071455.pdf].

11. Katz MH: Multivariable analysis: a primer for readers of medical research. Ann Intern Med 2003, 138:644-650.

doi:10.1186/ar3498

Cite this article as: Wener MH: Multiplex, megaplex, index, and complex: the present and future of laboratory diagnostics in rheumatology. Arthritis Research \& Therapy 2011, 13:134. 